\title{
Cognitive Radio for Ka Band Satellite Communications
}

\author{
Sina Maleki ${ }^{1}$, Symeon Chatzinotas ${ }^{1}$, Shree Krishna Sharma ${ }^{1}$, \\ Alessandro Guidotti ${ }^{2}$, Daniele Tarchi ${ }^{2}$, Alessandro Vanelli-Coralli ${ }^{2}$, \\ Wuchen Tang ${ }^{3}$, Barry A. Evans ${ }^{3}$, Joel Grotz ${ }^{4}$, Konstantinos Liolis ${ }^{5}$, Jens Krause ${ }^{5}$, and Nicolas Chuberre ${ }^{6}$, \\ ${ }^{1}$ Interdisciplinary Centre for Security, Reliability and Trust, University of Luxembourg \\ e-mail:\{sina.maleki, symeon.chatzinotas, shree.sharma\}@uni.lu \\ ${ }^{2}$ University of Bologna, Italy, e-mail:\{a.guidotti, daniele.tarchi, alessandro.vanelli\}@unibo.it \\ ${ }^{3}$ University of Surrey, UK, e-mail:\{w.tang, b.evans $\} @$ surrey.ac.uk \\ ${ }^{4}$ Newtec, Belgium, e-mail: joel.grotz@newtec.eu \\ ${ }^{5}$ SES, Luxembourg,e-mail:\{konstantinos.liolis, jens.krause\}@ses.com \\ ${ }^{6}$ Thales Alenia Space, France, e-mail: nicolas.chuberre@thalesaleniaspace.com
}

\begin{abstract}
The satellite communication data traffic is increasing dramatically over the coming years. High throughput multibeam satellite networks in Ka band are potentially able to accommodate the upcoming high data rate demands. However, there is only $500 \mathrm{MHz}$ of exclusive band for download and the same amount for upload. This spectrum shortage impose a barrier in order to satisfy the increasing demands. Cognitive satellite communication in Ka band is considered in this paper in order to potentially provide an additional $4.4 \mathrm{GHz}$ bandwidth for downlink and uplink fixed-satellite-services. In this way, it is expected that the problem of spectrum scarcity for future generation of satellite networks is alleviated to a great extent. The underlying scenarios and enabling techniques are discussed in detail, and finally we investigate the implementation issues related to the considered techniques.
\end{abstract}

\section{INTRODUCTION}

The demand for high data rates is increasing dramatically. At the same time, the current static frequency allocation regime does not provide adequate resources to satisfy the customers demand. Enabling cognitive access in the licensed bands seems a promising way to address this problem [1]. As in any wireless service, the demand for higher data rates obtained through satellite communications particularly in rural areas is increasing over the next years. The $\mathrm{Ka}$ band is shown to be a an appropriate band in order to deploy the future high throughput satellite services. However, there is only $500 \mathrm{MHz}$ of exclusive bandwidth available for such services. This limited spectrum is a barrier in order to accommodate the increasing satellite data traffic. Therefore, it becomes necessary for satellite communication to gain further spectrum access by dynamic utilization of the frequency bands assigned to other services without impeding operation of the incumbent users. In this way, cognitive radio appears as a promising concept to realize the dynamic spectrum utilization. In this paper, cognitive satellite communications are considered where the cognitive satellite networks coexist with the incumbent satellite or terrestrial networks [2]-[5]. Several scenarios are possible in order to enable cognitive satellite communication. These scenarios as well as their advantages and disadvantages are discussed and analyzed in [6] within the project Cognitive
Radio for Satellite Communications (CoRaSat). After rigorous analysis of each scenario, two downlink and an uplink scenarios in $\mathrm{Ka}$ band are selected as the most promising ones, through which $4.4 \mathrm{GHz}$ of bandwidth is considered for the cognitive satellite access.

The first scenario namely Scenario A considers a cognitive downlink access by fixed-satellite-service (FSS) terminals in the band $17.3 \mathrm{GHz}-17.7 \mathrm{GHz}$ where the incumbent users are broadcasting-satellite-service (BSS) feeder links. We follow by another downlink scenario called Scenario B where the cognitive FSS terminals dynamically utilize the band $17.7 \mathrm{GHz}$ 19.7 $\mathrm{GHz}$ with fixed-service (FS) microwave links as the incumbent users. In the last scenario which is called Scenario $\mathrm{C}$, a cognitive uplink setup is considered where the FSS terminals gain uplink access in the band $27.5 \mathrm{GHz}-29.5 \mathrm{GHz}$. The incumbent users in this band are FS links. Each scenario as well as the underlying enabling techniques are discussed in detail throughout this paper. The considered techniques include database assisted cognitive zones, spectrum sensing, and carrier-to-interference plus noise-ratio (CINR) estimation. As shall be defined later in detail, the cognitive zone is the geographical area around the FSS in downlink scenarios, and the FS in the uplink scenario, where the FSS terminals need to employ cognitive interference mitigation techniques.

The remainder of the paper is organized as follows. The Ka band multibeam system model followed by scenario definition are discussed in Section II. We carry on with outlining the required enabling techniques for each scenario in Section III. Section IV investigates the implementation issues, and finally we conclude the paper in Section V.

\section{System Model ANd SCEnARio Definition}

\section{A. Ka band Multibeam System}

Employing multibeam antennas, the new generation of satellite systems divide the coverage area into small beams in order to increase the spectrum efficiency as well as the quality of service. In this way, the available spectrum can be reused several times within the coverage area [7]. Multibeam satellites are particularly suitable for Ka band where potentially 
a very large bandwidth of spectrum is available for satellite communications.

Different satellite services such as BSS feeder links and FSS use Ka band for data transmission. The focus of this paper is on the FSS, and particularly on opportunistic utilization of those parts of $\mathrm{Ka}$ band where the incumbent user is not the FSS. In this paper, we are particularly interested in the frequency plans within $\mathrm{Ka}$ band as shown in Fig. 1. While the bands $19.7 \mathrm{GHz}-20.2 \mathrm{GHz}$ and $29.5 \mathrm{GHz}-30 \mathrm{GHz}$ are respectively assigned for the exclusive FSS downlink and uplink, the rest are to be shared with the incumbent users based on a cognitive frequency utilization paradigm. In the following subsections, we describe the scenarios that the FSS gains opportunistic access to the bands where the incumbent user is not the FSS.

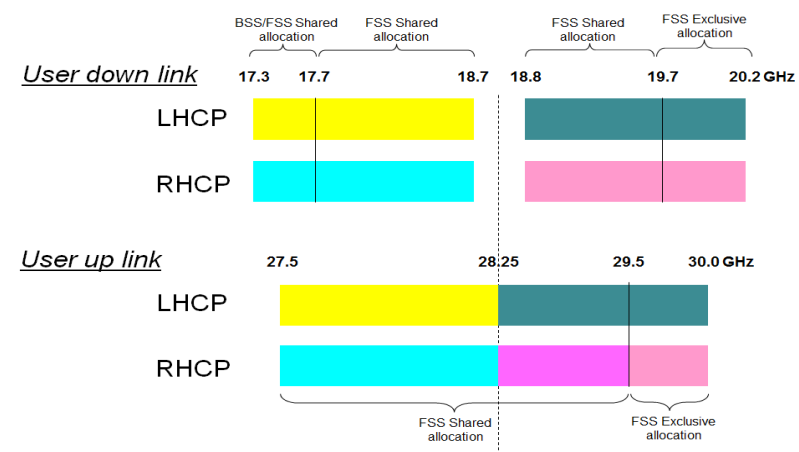

Fig. 1: Frequency plan 1 for CoRaSat. LHCP: Left Hand Circular Polarization, RHCP: Right Hand Circular Polarization

\section{B. Scenario A}

A cognitive FSS downlink scenario in $\mathrm{Ka}$ band $17.3 \mathrm{GHz}-$ 17.7 GHz is considered. The incumbent users are BSS feeder links that work in the uplink mode. Scenario A is depicted in Fig. 2. We can see that while the incumbent BSS feeder links are sufficiently protected in this scenario (due to GEO satellites orbital separation), the cognitive terminals need to protect themselves from the possible BSS feeder links interference.

\section{Scenario B}

As in Scenario A, the cognitive FSS terminal works in the downlink. The considered band in this scenario is $17.7 \mathrm{GHz}-$ 19.7 GHz, within Ka band. The incumbent users are terrestrial FS microwave links as shown in Fig. 3. Considering the power flux density regulations, the incumbent FS links are sufficiently protected in this scenario [6]. However, the FS transmitter can interfere the cognitive FSS terminals. Therefore, as in the previous scenario, the cognitive terminals should protect themselves against the incumbent interference.

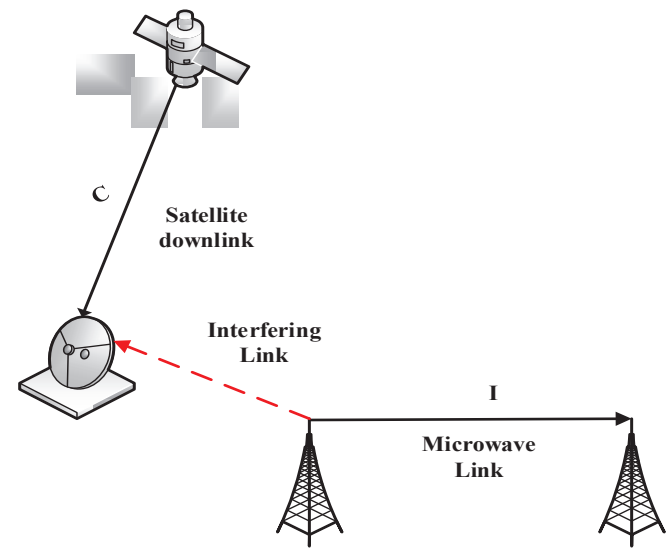

Fig. 3: Scenario B. C: Cognitive, I: Incumbent

\section{Scenario $C$}

We consider a cognitive FSS uplink scenario in Ka band 27.5 GHz-29.5 GHz. The incumbent users are again FS terrestrial microwave links. A schematic plan for this scenario is shown in Fig. 4. Unlike the previous scenarios, the incumbent users are generally not protected against the uplink FSS interference. Therefore, specific measures need to be taken into account in order to avoid any harmful interference to the incumbent users.

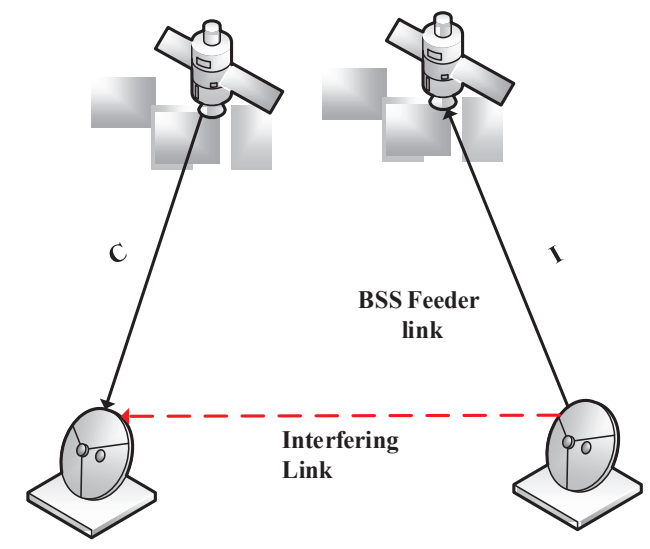

Fig. 2: Scenario A. C: Cognitive, I: Incumbent

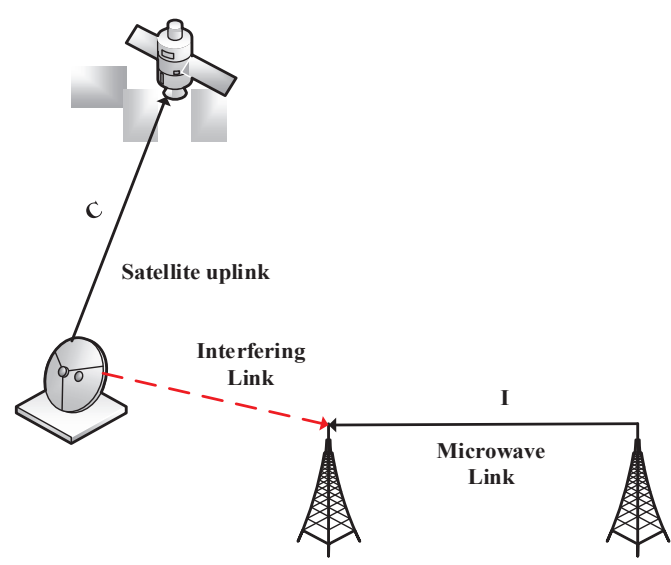

Fig. 4: Scenario C. C: Cognitive, I: Incumbent 
Each of the mentioned scenarios needs different techniques in order to facilitate cognitive access within the corresponding bands. In this paper, we focus on enabling techniques for each scenario.

\section{Cognitive Radio Techniques}

\section{A. Enabling Techniques for Scenario A}

As mentioned earlier, a cognitive downlink communication is considered in Scenario A. A FSS terminal coexists with the incumbent uplink BSS feeder links in the band $17.3 \mathrm{GHz}$ 17.7 GHz. Since the incumbent BSS feeder links work in the uplink mode, the interference from the cognitive satellite is limited, and the incumbent satellite is sufficiently protected. However, as shown in Fig. 2, the cognitive FSS terminal can be interfered with the uplink transmission of the incumbent BSS feeder links. To make sure that the cognitive terminal can resolve the received signal correctly, the received interference from the incumbent terminal should be below a specific threshold. Here, we propose three approaches in order to assure the QoS of the FSS terminal.

1) Cognitive Zone: A cognitive zone is defined as the area around the FSS terminals where the cognitive FSS terminal's interference from the incumbent terminals is above the nominated threshold. Therefore, within cognitive zone, the FSS terminals needs to employ cognitive interference mitigation techniques in order to gain spectrum access. This approach is particularly promising as there are only a few BSS feeder links per country. Further, it is relatively easy to obtain the information about their location, maximum power transmission, elevation angle, antenna pattern, and etc. In this way, a geographical area becomes open for FSS communication with no interference. Fig. 5 illustrates an example cognitive zone for a BSS database obtained from UK OFCOM. In this figure, we have only considered the free space path loss as the source of attenuation, however in reality, there are other sources of attenuation, e.g. scattering, geographical terrains, water vapor, buildings and so on, which reduce the interference significantly. In detail design of cognitive zones based on realistic interference modeling is a subject of ongoing research. Note that although the cognitive zone is one of the promising techniques to implement Scenario A, however, it can be combined with dynamic frequency assignment techniques, particularly when some of the frequency bands within the cognitive zone are available for the cognitive downlink access.

2) Dynamic Frequency Assignment with Spectrum Sensing: Dynamic frequency assignment with spectrum sensing is widely considered as the technique to design terrestrial cognitive radio systems. Cognitive radios listen to the spectrum in periodic time-slots and gain spectrum access as soon as a spectrum hole is discovered [8]. The sensing parameters are often designed to satisfy a specific detection performance in order to ensure the interference constraint as well as the quality of service (QoS) of the cognitive radios [9]. In Scenario $\mathrm{A}$, the incumbent BSS feeder links are protected from the harmful interference of the cognitive terminals, however, the FSS terminals can still employ spectrum sensing in order to avoid activity in those bands where the interference from the BSS feeder links is higher than a specific threshold, and particularly when the FSS terminal is within the cognitive

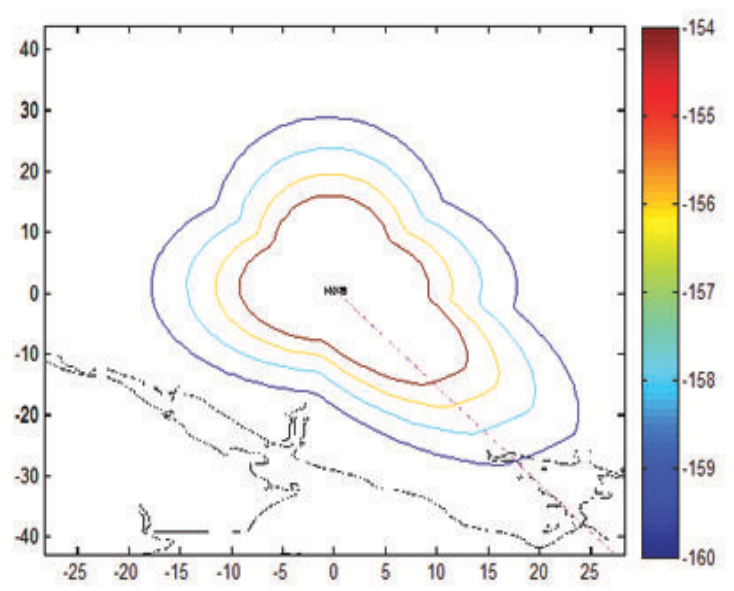

Fig. 5: Cognitive zone in Scenario A for UK BSS database based on free space path loss model (Line of Sight) [19].

zone. In this case, spectrum sensing boils down to power spectrum estimation. Power spectrum estimation is particularly promising as there are several techniques in order to estimate the power spectrum both above and below Nyquist rates [10][13]. This way, the FSS terminals can rapidly adapt their transmission frequencies to those with zero or acceptable BSS interference.

Considering the fact that when BSS feeder links are active, they are going to be active for very long periods of time, high frequency periodic spectrum sensing as in terrestrial cognitive systems is an overkill for Scenario A. Therefore, a virtually one-time sensing procedure in the beginning of the FSS activity seems to be sufficient. Further, note that since the FSS terminals are only interested in avoiding the BSS interference, missing detection of the BSS feeder links due to hidden terminal is not a problem for Scenario A. A one off sensing of the BSS interference at the proposed FSS terminal site e.g. by a horn scanning or dipole antenna in the azimuth plane might be sufficient [17]. However, this approach adds a high implementation cost to the system. In the following subsection, an alternative and continuous spectrum sensing technique based on CINR estimation is proposed.

3) Dynamic Frequency Assignment with CINR estimation: The FSS terminal is able to control the quality of the received signal with CINR estimation. If the estimated CINR is not satisfactory, then the gateway has to stop data transmission through this carrier or move to another carrier where an acceptable received CINR can be achieved. While this technique is easy to implement, a problem arises when the low CINR is not due to the BSS interference but due to the rain fading. To solve this issue, we exploit the control channel signaling towards the FSS terminal which can be decoded more easily due to larger CINR margins. The control channel frames are encoded with using the lowest modcod (QPSK 1/2), and thus allowing larger CINR margins. First, the satellite downlink frame is detected, decoded and subtracted. The remaining signal power is noise and the possible BSS interference. The energy of the remaining part is then calculated and possible received interference from 
the BSS feeder links is estimated assuming knowledge of the noise variance. Denoting $\mathbb{I}_{\mathrm{BSS}}$ to be the received interference of the BSS feeder links, $\mathcal{H}_{0}$ as the case where the BSS feeder links interference are harmful and $\mathcal{H}_{1}$ as the opposite case, in order to decide among $\mathcal{H}_{0}$ and $\mathcal{H}_{1}$, we employ a decision testing problem as follows

$$
\mathbb{I}_{\text {BSS }} \stackrel{\mathcal{H}_{1}}{\underset{\mathcal{H}_{0}}{\gtrless}} \gamma .
$$

where $\gamma$ is the maximum allowable interference. The outcome of (1) leads to a spectrum access or spectrum handoff.

\section{B. Enabling Techniques for Scenario B}

As mentioned before, in Scenario B the cognitive FSS terminal works in the downlink and coexists with terrestrial FS microwave links in the $17.7 \mathrm{GHz}-19.7 \mathrm{GHz}$, within $\mathrm{Ka}$ band. As for the implementation of the cognitive link, observations similar to those made for Scenario A still hold. There is no interference from the cognitive satellite towards the incumbent FS microwave link, while the cognitive FSS terminal might actually be interfered by the incumbent transmissions as shown in Fig. 3. With respect to Scenario A, the additional challenge is related to the different geometry of the incumbent and cognitive links. The following technical approaches are proposed to guarantee the desired QoS at the cognitive FSS terminal.

1) Cognitive Zone: As in Scenario A, a cognitive zone around the FSS terminal is determined so as to the cognitive FSS terminal does not receive harmful interference from the incumbent users. It shall be noted that although the cognitive zone is one of the promising techniques to implement Scenario $\mathrm{B}$, a downlink carrier handover might be required in case the desired QoS cannot be guaranteed at the cognitive terminal within the cognitive zone. In particular, the choice of the new carrier frequency may rely on a combination of data provided by a database and spectrum sensing.

2) Geolocation Database: Satellite ground stations can potentially employ spectrum sensing in order to determine whether a FS transmitter is present or not. Assuming that the sensitivity of spectrum sensing is enough to detect the maximum interference level that the satellite ground station can tolerate, the decision can be based solely on the spectrum sensing observation. Additionally, the locations of the FS transmitters and the satellite ground stations are fixed along with the transmission power of the FS transmitter, and thus a geolocation database approach can provide a reliable solution [14]-[16] to assist determining the cognitive zones and spectrum sensing. All this information is known and can be centralized in a database that the satellite ground station can query in order to determine whether a specific band is available or not, without the need of using spectrum sensing. Alternatively, a combination of database and spectrum sensing can be implemented in order to reach a more reliable decision. Sometimes database information is not accurate, e.g. a part of spectrum is announced by the database as occupied, but this information is outdated and in reality indeed the spectrum is available for cognitive access. In such situations, spectrum sensing can be employed in order to authenticate the data base and to search spectrum holes in the FS allocations which are free.
3) Dynamic Frequency Assignment with Spectrum Sensing/CINR Estimation: Given the similarity between Scenario A and Scenario B, dynamic frequency assignment techniques based on spectrum sensing or CINR estimation can be used. As for spectrum sensing, in this scenario the incumbent FS microwave links are protected from harmful interference of the cognitive terminals, and thus spectrum sensing techniques can be employed at the cognitive terminals within the cognitive zone in order to determine the bands where the interference received by the incumbent transmitters is below a predefined threshold. Thus, power estimation provides a valuable solution for Scenario B as well [17]. Moreover, the same observation on the activity of the BSS feeder links holds for the FS microwave transmitters, and thus a one-time sensing procedure seems to suffice, and the hidden terminal is not an issue in this scenario as well. However, as in Scenario A, an azimuth steering horn antenna or dipole can be employed to measure the FS interference at the site of the terminal [17]. A limiting factor to measure the interference can be the noise variance uncertainty due to noise variance estimation error [18]. Fig 6 depicts the required number of observation samples in order to successfully measure the received interference for a range of FS interference to noise ratios (I/N) and noise uncertainties. Further, we consider sensing at the dish as well as an additional dipole antenna. It is shown that employing an additional dipole antenna, the interference can be successfully detected for $I / N \geq-10 \mathrm{~dB}$ within finite time. $\mathrm{I} / \mathrm{N}$ of $-10 \mathrm{~dB}$ is considered by the FSS producers as well as the regulatory bodies as the worst-case long-term interference constraint. In the same way, we can obtain similar results for Scenario A. Therefore, employing energy detection at an additional dipole antenna yields reliable sensing results for cognitive downlink satellite communications.

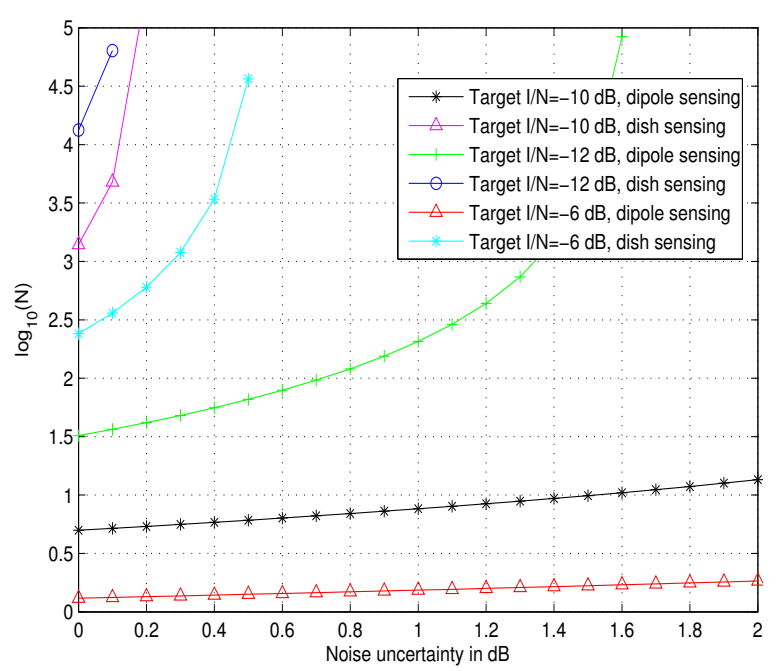

Fig. 6: Required number of samples for successful harmful interference detection at the FSS terminal versus noise variance uncertainty [17].

As in Scenario A, the cognitive terminal can control the quality of the received signal through CINR estimation as well, which allows to identify the most suitable carrier for the required service. Again some issues may arise in case of rain fading. However, the same solution proposed in the previous 
section can be adopted: once the satellite downlink frame is detected, decoded and subtracted, the remaining signal power is given by noise and the possible incumbent interference. Thus, assuming that the noise variance is known, the decision testing problem provided in (1) can be applied.

\section{Enabling Techniques for Scenario $C$}

Scenario C considers the co-existence of terrestrial FS microwave links and FSS uplinks operating in $27.5 \mathrm{GHz}$ $29.5 \mathrm{GHz}$ of $\mathrm{Ka}$ band. The FS microwave link is the incumbent system and the FSS uplink is the cognitive system. As an uplink symmetric Scenario of Scenario B, in Scenario C, there is no interference from the incumbent FS microwave link towards the cognitive satellite, while the potential interference is from the cognitive FSS transmitting terminal towards the incumbent FS microwave link as shown in Fig. 4. To provide sufficient protection for the FS microwave link, FSS gateways are always coordinated but there are only few of these. In the case of small FSS user terminals, a full coordination scheme becomes complex and thus we could search for a rapid coordination scheme that can be used to investigate the possibility of spectrum sharing in this scenario. The following technical approaches are also considered.

1) Cognitive Zone: In this scenario, a cognitive zone around the FS microwave transmitter is determined beyond which, the cognitive FSS terminal is allowed to share the bands with the incumbent terminals without producing intolerable interference. This approach would also be promising here if the information about the FS microwave links is easy to obtain. There are large number of FS links in this band and in some countries where spectrum sales have occurred, it is impossible to obtain the actual data. But in countries where this information is available, it may be that only a few FS links will be potential interfere to the cognitive FSS terminal.

2) Geolocation Database: Similar to Scenario B, a geolocation database approach would also apply here. If all the relevant information is known and can be centralized in a database, the cognitive FSS terminals can query in order to determine whether a specific band used by the incumbent FS microwave link is available or not, without the need of using spectrum sensing. Or it is possible that the sensing can be used on a longer time period to authenticate databases if the accuracy of the local database can not be guaranteed. However, the feasibility of this approach also depends on the availability of the database on a local country basis.

3) Dynamic Frequency Assignment with Spectrum Sensing: Dynamic Frequency Assignment techniques based on spectrum sensing may not be easy to implement in Scenario C, because the interference is from the cognitive FSS terminal to the FS microwave link, and the FSS terminals are in the uplink spectrum band. In this case, the sensing in the actual system would have to be done in the FS sector, which is practically hard in the sense that the incumbent user has no reason to adapt its infrastructure with respect to the cognitive system. However there may be only a few FS links affected, and thus such an approach could be implemented by some collaboration with the cognitive users.

\section{IMPLEMENTATION ISSUES}

The possible system level design of the cognitive satellite systems considering the regulatory constraints as well as the QoS was considered in the previous subsections. In practice, there are some implementation issues which also needs to be taken into account. The implementation aspects of the selected scenarios is considered in this subsection.

The terminal in the context of the cognitive link to the satellite and in presence of the incumbent terrestrial links has to cope with different interference situations leading to a large range of interference powers and potential protection ratios. The terminal antenna systems are very directive (parabolic antennas) and the terrestrial and satellite systems have very different link power densities. Therefore the link budget difference between the terrestrial incumbent system and the cognitive satellite system can be very significant and lead to problems in the cognitive link that are only related to the presence of the high power signal. It is assumed that specific countermeasures are required in the front-end of the cognitive satellite terminal. The following interference implementation problems in this context are considered:

- Scenario A: the proximity of the BSS feeder link and the cognitive FSS terminal can lead to potentially also high power interference into the cognitive link reception.

- Scenario B: the cognitive terminal receives in the same band as the terrestrial FS links. The resulting interference at the cognitive satellite terminal input can range up to high power in case the satellite reception aligns with the FS transmission and is close enough.

- Scenario C: the detection and sensing of the FS link in presence of the cognitive FSS transmission can be a problem in case the sensor is linked to the FSS parabolic satellite antenna. Indeed the situation of an FSS transmission aligned with a terrestrial FS reception can possibly not be sensed ideally. Therefore the usage of a specific sensor in combination with the terrestrial antenna is considered.

For the considered scenarios we see that there is potentially a need for specific blocking filters in the low noise block (LNB) to counteract the potentially high interference in case of scenarios A and B. In addition there is likely a need for an optional omni-directional sensor fixed on the parabolic reflector of the cognitive satellite terminal. With these two aspects, it is assumed that an adapted front-end to the requirements of the cognitive system aspects may be required. This is further considered as an option in the context of the CoRaSat project with further quantitative analysis

\section{CONCLUSIONS}

Cognitive satellite communication in Ka band was considered in this paper. Three scenarios were introduced as the most promising ones in this band in order to provide high data rate FSS. It was shown that these scenarios can potentially bring up $4.4 \mathrm{GHz}$ of extra bandwidth. The underlying enabling techniques including the cognitive zone, database, spectrum sensing, and CINR estimation were discussed. It was shown that beyond cognitive zone, no extra interference mitigation 


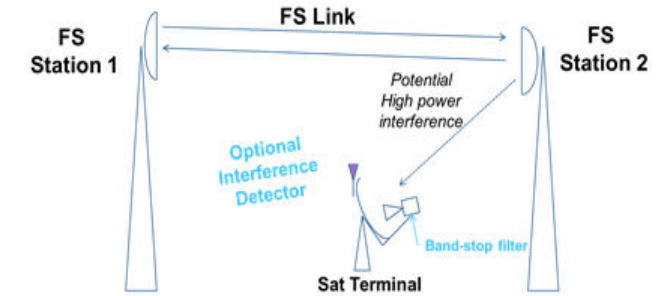

FS $1 \rightarrow 2$ : Difficult to detect $\rightarrow$ Interference detector antenna on reflector FS $2 \rightarrow 1$ : Potential high power interference $\rightarrow$ Band-stop filter in LNB

Fig. 7: Adopted front-end for the CoRaSat user terminal.

techniques is required at the FSS terminal. However, within cognitive zone, the FSS terminals need to employ cognitive techniques in order to tackle the imposed interference or avoid imposing interference. We further discussed about the implementation issues and proposed adding an additional antenna to the dish in order to improve the sensing reliability. The additional antenna can be also used for beamforming purposes in order to mitigate the received interference, or produce a null towards the FS receiver in the uplink scenario.

Note that other techniques such as beamforming in order to improve the sensing result and CINR, and spectrum exploitation techniques, e.g. dynamic carrier allocation, were not discussed in this paper. Investigation of these techniques and numerical performance analysis of the scenarios introduced in this paper are our ongoing focus of research.

\section{ACKNOWLEDGMENT}

The authors would like to thank the European Commission (EC) for their co-funding support through the CoRaSat project (FP7 ICT STREP Grant Agreement n. 316779).

\section{REFERENCES}

[1] D. Cabric, S.M. Mishra, D. Willkomm, R. Brodersen, and A. Wolisz, "A cognitive radio approach for usage of virtual unlicensed spectrum," 14th IST Mobile and Wireless Communications Summit. 2005.

[2] K. Liolis, G. Schlueter, J. Krause, F. Zimmer, L. Combelles, J. Grotz, S. Chatzinotas, B. Evans, A. Guidotti, D. Tarchi, and A. Vanelli-Coralli, "Cognitive radio scenarios for satellite communications: The CoRaSat approach," Future Network and Mobile Summit (FutureNetworkSummit), 2013 , vol., no., pp.1,10, 3-5 July 2013.

[3] S.K. Sharma, S. Chatzinotas, and B. Ottersten, "Cognitive Radio Techniques for Satellite Communication Systems," 2013 IEEE 78th Vehicular Technology Conference (VTC Fall), pp.1-5, 2-5 Sept. 2013.

[4] S. Kandeepan, L. De Nardis, M. Di Benedetto, A. Guidotti, and G.E. Corazza, "Cognitive Satellite Terrestrial Radios," 2010 IEEE Global Telecommunications Conference (GLOBECOM 2010), pp.1,6, 6-10 Dec. 2010 .

[5] M. Hoyhtya, J. Kyrolainen, A. Hulkkonen, J. Ylitalo, and A. Roivainen, "Application of cognitive radio techniques to satellite communication," IEEE International Symposium on Dynamic Spectrum Access Networks (DYSPAN), 2012, pp.540,551, 16-19 Oct. 2012.

[6] G. Schlueter et al., "Deliverable D2.3: Scenarios definition and selection", EU FP7 CoRaSat Project, Oct. 2012. available online on https://docs.google.com/file/d/0BzUS3rOEUdTwZ3ZxSjBWb0ptYzQ/edit

[7] G. Maral, and M. Bousquet, "Satellite communications systems", John Wiley \& Sons 2009.

[8] D. Cabric, I.D. O’Donnell, M.S.-W Chen, and R.W. Brodersen, "Spectrum sharing radios," Circuits and Systems Magazine, IEEE, vol.6, no.2, pp.30,45, 2006.
[9] E. Axell, G. Leus, E. Larsson, H.V. Poor, "Spectrum Sensing for Cognitive Radio : State-of-the-Art and Recent Advances," IEEE Signal Processing Magazine, vol.29, no.3, pp.101,116, May 2012.

[10] D.D. Ariananda, and G. Leus, "Compressive wideband power spectrum estimation”, IEEE Transactions on Signal Processing, 60(9):47754789, September 2012.

[11] R. Arroyo-Valles, S. Maleki, and G. Leus, "Distributed wideband spectrum sensing for cognitive radio networks", 2014 IEEE International Conference on Acoustics, Speech, and Signal Processing, ICASSP 2014, Florence, Italy.

[12] P. Stoica, and R.L. Moses, "Spectral analysis of signals", Upper Saddle River, NJ: Pearson/Prentice Hall, 2005.

[13] J. Li, and P. Stoica, "An adaptive filtering approach to spectral estimation and SAR imaging," IEEE Transactions on Signal Processing, vol.44, no.6, pp.1469,1484, Jun 1996.

[14] Ofcom UK, "Digital Dividend: Geolocation for Cognitive Access; a discussion on using geolocation to enable license-exempt access to the interleaved spectrum," November 2009.

[15] Ofcom UK, 'Implementing Geolocation,” November 2010.

[16] ECC Report 186, 'Technical and operational requirements for the operation of white space devices under geo-location approach," January 2013.

[17] S.K. Sharma, S. Maleki, S. Chatzinotas, J. Grotz, and B. Ottersten, "Implementation issues of cognitive radio techniques for Ka-band (17.7-19.7 GHz) SatComs", 7th Advanced Satellite Multimedia Systems Conference, ASMS 2014, Livorno, Italy.

[18] R. Tandra, and A. Sahai, "SNR Walls for Signal Detection," IEEE Journal of Selected Topics in Signal Processing, vol.2, no.1, pp.4,17, Feb. 2008.

[19] N. Chuberre, et al. "FP7 PROJECT CoRaSat intermediate results and standardization strategy", $\mathrm{EuCnC} 2014$. 